\title{
Management strategies for patients with subclinical hypothyroidism: a protocol for an umbrella review
}

\author{
Brenda S. Bauer ${ }^{*}$, Amaya Azcoaga-Lorenzo, Utkarsh Agrawal and Colin McCowan
}

\begin{abstract}
Background: Subclinical hypothyroidism is a thyroid disorder diagnosed from the laboratory blood test results of otherwise asymptomatic patients. It has been associated with poor cardiovascular outcomes, mortality and progression to overt thyroid hormone deficiency. Current guidelines on the management of subclinical hypothyroidism differ because of conflicting evidence on long-term treatment benefits. Even though there are several existing systematic reviews on its clinical outcomes, no definitive conclusion has been reached yet. As such, a new synthesis could help provide more insight and consensus on this topic. To this purpose, this umbrella review will evaluate and synthesise current evidence on the long-term clinical outcomes of the different management strategies for subclinical hypothyroidism.
\end{abstract}

Methods: This is a protocol for an umbrella review on the management strategies for subclinical hypothyroidism. We will conduct literature searches in multiple electronic databases (from inception onwards), namely MEDLINE, EMBASE, Scopus, Web of Science, Cochrane Database of Systematic Reviews, JBI Evidence Synthesis, Epistemonikos database, PDQ Evidence and the PROSPERO register. There will be no restriction on the date or language of publication. Additional material will be identified through grey literature searches and citation chaining. Review inclusion criteria will be patients with subclinical hypothyroidism, receiving treatment or monitoring, no restrictions on the comparators used and with cardiovascular events, frailty fractures, quality of life and all-cause mortality as primary outcomes of interest. Two reviewers will independently screen all citations, full-text articles and abstract data on a pre-piloted form in duplicate. Methodological quality (or bias) of included studies will be appraised using AMSTAR-2. Any conflicts that arise will be resolved through discussion or involving a third reviewer. A narrative synthesis will be provided with information presented in the main text and tables to summarise and explain the characteristics and findings of the included reviews. Even so, it is not expected that a meta-analysis will be performed due to review variability. Study limitations and methodological quality assessments will also be reported to provide context for the overall summary of evidence.

Discussion: This review will provide a comprehensive summary of the effects of the pharmacological and non-pharmacological management of subclinical hypothyroidism on specific long-term clinical outcomes. It is anticipated that the findings of this umbrella review will aid in the development of consensus-based clinical recommendations for subclinical hypothyroidism, as well as highlight areas for future research. Review findings will be disseminated primarily through peer-reviewed publications.

\footnotetext{
${ }^{*}$ Correspondence: bsb1@st-andrews.ac.uk
}

School of Medicine, University of St Andrews, North Haugh, St

Andrews KY16 9TF, UK original author(s) and the source, provide a link to the Creative Commons licence, and indicate if changes were made. The images or other third party material in this article are included in the article's Creative Commons licence, unless indicated otherwise in a credit line to the material. If material is not included in the article's Creative Commons licence and your intended use is not permitted by statutory regulation or exceeds the permitted use, you will need to obtain permission directly from the copyright holder. To view a copy of this licence, visit http://creativecommons.org/licenses/by/4.0/. The Creative Commons Public Domain Dedication waiver (http://creativeco $\mathrm{mmons}$.org/publicdomain/zero/1.0/) applies to the data made available in this article, unless otherwise stated in a credit line to the data. 
Systematic review registration: PROSPERO CRD42021235172

Keywords: Subclinical hypothyroidism, Levothyroxine, Systematic review, Review of systematic reviews, Umbrella review

\section{Background}

Subclinical hypothyroidism $(\mathrm{SCH})$ is a disorder of the thyroid gland in which blood levels of free circulating hormones are normal, but those of thyroid-stimulating hormone (TSH)-which stimulates thyroid hormone production-are elevated. It can only be diagnosed through laboratory tests, and diagnosed patients are typically asymptomatic $[1,2]$. As such, the detection of $\mathrm{SCH}$ is often incidental [3, 4], and in approximately 2 to $5 \%$ of patients, $\mathrm{SCH}$ has been found to progress to overt hypothyroidism [2]. SCH has also been linked to an increased risk of cardiovascular disease and mortality [5, 6], frailty fractures [7], cognitive dysfunction, anxiety and depression [8]. Crucially, however, these associations are based on differing conclusions from observational studies and small randomised trials with relatively brief follow-up periods [9].

$\mathrm{SCH}$ is treated through the replacement of thyroid hormone using the drug levothyroxine [10]. Even so, the decision to begin replacement therapy has long been controversial because of conflicting findings on whether treatment is beneficial for long-term outcomes [1, 2]. Recently published guidelines on the management of $\mathrm{SCH}$ differ in their recommendations, as a result. One evidence-based guideline recommends applying a TSH threshold of $10 \mathrm{mU} / \mathrm{L}$ for prescribing levothyroxine because of potential long-term benefits such as cardiovascular outcomes and symptom improvement [11, 12]. On the other hand, Bekkering et al. [13] considered a systematic review of 21 trials that found minimal to no evidence of clinical benefit from replacing thyroid hormones in $\mathrm{SCH}$ [14]. In response, a strong recommendation was issued against treatment for most adult patients, except patients with TSH levels greater than $20 \mathrm{mIU} / \mathrm{L}$ and pregnant women [13].

It is widely acknowledged that inadequate research has been conducted on the long-term clinical outcomes of managing $\mathrm{SCH}$, especially as inconsistencies remain in the findings of the studies that have been performed to date $[1,9,15-17]$. Since thyroid hormone replacement is a lifelong treatment, it is vital to investigate how levothyroxine affects health in subclinical disease. Equally important are the clinical effects of follow-up with no treatment-for patients who do not meet treatment thresholds, for example-in determining the optimal timing of treatment, as well as the suitability of certain patient groups to receive treatment.
The umbrella review approach is well-suited to the synthesis of a body of contentious evidence, as it allows for a rigorous and systematic assessment of the literature [18]. We will employ this methodology to summarise and compare systematic reviews of various clinical outcomes of the management strategies of subclinical hypothyroidism, which may be either to prescribe treatment or to monitor the patient with no pharmacological intervention. Specifically, the review questions are:

Q1: What is the impact of levothyroxine treatment on long-term clinical outcomes for patients with subclinical hypothyroidism?

Q2: What is the impact of follow-up without treatment on long-term clinical outcomes for patients with subclinical hypothyroidism?

\section{Methods}

\section{Protocol development}

This protocol was registered in the PROSPERO register [19] as CRD42021235172. The methods described below are based mainly on the 'Umbrella Reviews' chapter of the JBI Manual for Evidence Synthesis [20], though some elements-protocol length, referencing style, critical appraisal and data collection tools, in particular-have been adapted for our purposes. The Preferred Reporting Items for Systematic Review and Meta-Analysis Protocols (PRISMA-P) guidelines [21, 22] have been followed in reporting this protocol, for which a completed checklist is provided as an additional file [see Additional file 1].

\section{Inclusion criteria}

It is anticipated that all the systematic reviews obtained for this review will have clearly defined inclusion and exclusion criteria, in keeping with systematic review norms and guidelines. Therefore, it will be possible to apply the following criteria while selecting the relevant literature. A summary of the screening criteria is presented in Table 1.

\section{Participants}

The population of interest is restricted to patients diagnosed with subclinical hypothyroidism, regardless of age, setting and the country in which the studies took place. Reviews relating solely to pregnant women, children and adolescents will be excluded because these are special patient groups with additional clinical considerations. 
Table 1 Review inclusion and exclusion criteria

\begin{tabular}{|c|c|c|}
\hline & Inclusion criteria & Exclusion criteria \\
\hline Participants & $\begin{array}{l}\text { Patients diagnosed with subclinical hypothyroidism, no demo- } \\
\text { graphic or etiological restrictions }\end{array}$ & $\begin{array}{l}\text { Pregnant women } \\
\text { Children and adolescents } \\
\text { Diagnosis of overt hypothyroidism }\end{array}$ \\
\hline Intervention & $\begin{array}{l}\text { Management of } \mathrm{SCH} \text {, i.e. treatment using levothyroxine or follow- } \\
\text { up without treatment }\end{array}$ & No reported treatment status \\
\hline Comparator & No restriction on comparison or control groups & \\
\hline Outcomes & $\begin{array}{l}\text { Cardiovascular outcomes } \\
\text { Cerebrovascular outcomes } \\
\text { Quality of life measures } \\
\text { Frailty fractures } \\
\text { All-cause mortality } \\
\text { Other reported outcomes (secondary) }\end{array}$ & No reporting of any of the primary outcomes of interest \\
\hline Study design & $\begin{array}{l}\text { Quantitative systematic reviews and meta-analyses of empirical } \\
\text { research }\end{array}$ & $\begin{array}{l}\text { Any other study types (e.g. narrative reviews, scoping } \\
\text { reviews, qualitative syntheses) }\end{array}$ \\
\hline Review characteristics & $\begin{array}{l}\text { Articles in any language } \\
\text { Any period of study or date of publication }\end{array}$ & \\
\hline
\end{tabular}

\section{Intervention}

Inclusion is restricted to systematic reviews of studies involving the management of $\mathrm{SCH}$, whether (i) using levothyroxine for treatment or (ii) follow-up with no treatment. Studies that do not report the treatment status of participants will not be included.

\section{Comparator}

Any comparison groups will be eligible for inclusion, depending on whether one was used in the synthesis. Therefore, reviews that compare the effects of treatment against no treatment will be included, as well as those that report findings from only one of the two strategies.

\section{Outcomes}

The primary clinical outcomes of interest are cardiovascular (e.g. heart disease, heart failure, peripheral vascular disease), cerebrovascular (i.e. stroke), quality of life measures (e.g. Underactive Thyroid-Dependent Quality of Life score, Short-Form 36, Thyroid-Related Qualityof-Life Patient-Reported Outcome Measure), frailty fractures and all-cause mortality. Secondary outcomes (e.g. improvements in clinical symptoms, cognitive dysfunction) will also be included if reported in addition to the above.

\section{Study design}

Only quantitative systematic reviews and meta-analyses of empirical studies will be eligible for inclusion, regardless of whether the studies were randomised clinical trials or observational. Narrative and scoping reviews, as well as purely qualitative reviews, will be excluded during study selection. Any systematic reviews that include theoretical or opinion articles will also be considered ineligible.

Primary studies will not be considered, even when gaps are identified in the evidence within included systematic reviews.

\section{Review characteristics}

There will be no limitations on the year of publication or study period to allow for temporal comparisons in study findings. Publications in languages other than English will be included in the first instance; if translation is not possible, they will be excluded, but their details reported.

\section{Information sources and search strategy}

Comprehensive searches will be carried out on multiple electronic databases: MEDLINE, EMBASE, Scopus, Web of Science, Cochrane Database of Systematic Reviews, JBI Evidence Synthesis, Epistemonikos database, PDQ Evidence and the PROSPERO register, from inception onwards. There will be no additional filters based on the date or language of publication.

We will use controlled vocabularies and search terms directly related to the review questions such as 'treatment', 'levothyroxine' and 'subclinical hypothyroidism' which will be modified, as needed, to account for database-specific differences. Search filters will be applied to retrieve only systematic reviews. The MEDLINE search strategy, developed with the assistance of an academic librarian, is shown in Additional file 2.

The reference lists of selected reviews will also be checked for eligible syntheses (backward citation chaining) and Google Scholar used for forward citation chaining. A search will also be performed for grey literature, 
on the WorldCat and Open Grey databases and Internet search engines.

These searches will be updated in the later stages of the review (i.e. during data synthesis) to identify any relevant systematic reviews that will have been published in the interim.

\section{Study selection}

All the references retrieved from the searches will be imported to EndNote X9 [23] to remove duplicate records. The remaining citations will then be imported to Covidence [24] and screened independently by a set of two reviewers in duplicate-first by titles and abstractsagainst the inclusion and exclusion criteria described above. In situations where it is impossible to identify inclusion from the title and abstract alone, these articles will progress to full-text review.

Afterwards, articles that pass through the initial screening will be obtained and read in full to determine their eligibility for inclusion. Any disagreements in study selection will be resolved through discussion or the involvement of a third reviewer to reach a consensus. Updated systematic reviews will be included but treated as a single study to prevent duplication during data extraction. All decisions at this stage will be recorded and presented in a PRISMA flow diagram in subsequent reports.

\section{Data extraction}

A pair of reviewers working independently will use a standardised, pre-piloted form to extract data in duplicate. Specifically, data will be collected on first author, year of publication, reported a protocol, objective(s), reported strategies to search literature, number of databases searched and date of last search, any restrictions (e.g. language, geographic or date), inclusion/exclusion criteria, intervention(s) of interest and comparators, patient population, main outcomes of interest, type of study designs included (e.g. randomised controlled trials, observational studies or both), number of included studies, number of studies reporting data for meta-analyses, effect metric(s) reported (e.g. risk ratio), methods to assess study risk of bias, statistical methods to combine studies, summary meta-analytic estimates including heterogeneity measures, additional analyses (e.g. subgroup analysis or sensitivity analysis), metabias assessment (e.g. publication bias across studies), funding source and conflicts of interest. Where presented, data on the Grading of Recommendations, Assessment, Development and Evaluation (GRADE) rating for individual systematic reviews will also be collected. A complete list of fields to be extracted from included reviews is included in Additional File 3.
Disagreements arising from data extracts will be resolved by discussion with a third reviewer to reach a consensus. Where necessary, review authors will be contacted for further information on incomplete or missing data.

\section{Quality assessment}

The critical appraisal of all selected systematic reviews will be conducted in tandem with data extraction, using the AMSTAR (A MeaSurement Tool to Assess systematic Reviews) tool. This checklist was designed to assess the methodological quality of systematic reviews of randomised trials [25] and is currently in its second version, AMSTAR-2 [see Additional File 4]. In recognition of the increasing number of systematic reviews incorporating data from non-randomised and observational studies, the original checklist was updated, published and subsequently validated [26, 27]. Syntheses are judged on 16 domains, including the suitability of the research question and inclusion criteria, the search strategy, the characteristics and critical appraisal of included studies and publication bias. Most domains are rated either 'Yes' or 'No' though some have the additional option of 'Partial Yes'.

Discrepancies in the independent assessments made by each pair of reviewers will be resolved by discussion with a third reviewer to reach a consensus. The results of the quality assessments will be applied in the overall synthesis and presentation of findings so that it will also be possible to compare the included reviews by methodological quality. However, the primary studies from included systematic reviews will not be evaluated individually.

\section{Data synthesis}

Review findings will be synthesised narratively, as it is anticipated that there will be several differences in inclusion criteria, methods of synthesis and outcome measures. Overall outcome measures will be presented in tabular form, accompanied by detailed descriptions of review characteristics and quality assessments.

If there are sufficient data from the included systematic reviews, patient characteristics (e.g. age, sex) and methodological differences (e.g. search strategies, definitions of clinical outcomes) will be used to stratify the findings, to allow for further comparisons in the management options for $\mathrm{SCH}$ based on these criteria.

There is a considerable burden involved in performing a meta-analysis of existing systematic reviews, given the likelihood of primary studies being counted more than once [28]. This is because of the complexity of taking each review apart and then combining the results of several individual studies, many of which are likely to have different review questions and inclusion criteria. As such, 
it is anticipated that a meta-synthesis of included metaanalyses will not be performed; key statistical data will only be summarised.

\section{Confidence in cumulative evidence}

The GRADE ratings described within the included systematic reviews will be reported in this umbrella review. However, it is anticipated that not all studies will report these measures, especially older syntheses published prior to the first GRADE guidelines [29]. For such reviews, no new GRADE assessments will be conducted because they involve an assessment of primary studies. As such, this is beyond the scope of this umbrella review.

\section{Discussion}

This is a protocol outlining the processes through which an umbrella review will be performed. It is anticipated that this review of systematic reviews will be useful in summarising and comparing the syntheses of evidence on the management of $\mathrm{SCH}$. As such, its findings may either aid in the development of, or reinforce future evidence-based clinical guidelines. Furthermore, the review will be useful for the identification of any potential biases or gaps that could explain the contradictions in the literature on this topic. Knowledge gaps identified in the literature can also inform future studies and systematic reviews.

The key strength of this overview will be to provide a comprehensive summary of current evidence on the management of $\mathrm{SCH}$ through the application of robust and established methods to source, select, appraise and synthesise existing systematic reviews. This information will be of interest to researchers, clinicians and patients with $\mathrm{SCH}$ seeking a high-level overview of the evidence; this will be the first umbrella review on this topic, to the authors' knowledge.

This type of evidence synthesis - the umbrella reviewthough useful, is also subject to several limitations. First, inclusion in this review is restricted to systematic reviews, but additional empirical studies on the same topic are likely to have since been published. These new findings would, therefore, not be captured in the scope of this secondary synthesis. For this reason, all searches will be updated at least once, towards completion of the review.

Another potential challenge when applying metareview methodology is overlap in primary research. Study results included in more than one systematic review can cause misleading findings through a multiplier effect because a specified set of findings would be counted more than once. Therefore, a crucial element of data extraction and the subsequent synthesis will be to identify all primary studies and report all instances of overlap.

A third limitation is the differences in inclusion criteria between included studies that impede more quantitative forms of synthesis when conducting an overview. However, given the aim of this review of systematic reviews to collate and summarise all the synthesised literature on the clinical management of $\mathrm{SCH}$, a descriptive and tabular presentation of findings should suffice.

\section{Protocol amendments}

Any amendments to this protocol in the carrying out of this umbrella review will be documented and reported in both the PROSPERO register and any subsequent publications.

\section{Dissemination plans}

The findings of this umbrella review will be disseminated through publication in peer-reviewed journals, via social media networks and relevant conferences.

\section{Abbreviations}

AMSTAR: A MeaSurement Tool to Assess systematic Reviews; GRADE: Grading of Recommendations, Assessment, Development and Evaluation; IU: International units; PICO: Population, Intervention, Comparator and Outcomes; PRISMA: Preferred Reporting Items for Systematic review and Meta-Analyses; PRISMA-P: Preferred Reporting Items for Systematic Review and Meta-Analysis Protocols; SCH: Subclinical hypothyroidism; TFT: Thyroid Function Test; TSH:

Thyroid-stimulating hormone.

\section{Supplementary Information}

The online version contains supplementary material available at https://doi. org/10.1186/s13643-021-01842-y.

Additional file 1. PRISMA-P checklist.

Additional file 2. MEDLINE search strategy.

Additional file 3. Data extraction template.

Additional file 4. AMSTAR-2 checklist.

\section{Acknowledgements}

We thank Vicki Cormie, a senior librarian at the University of St Andrews, for her assistance in developing the search strategy for this review.

\section{Authors' contributions}

BSB led the design of the protocol, search strategy, drafting and revising of the manuscript. CM, AA and UA provided oversight and critically reviewed and revised the manuscript. All the authors read and approved the final manuscript. CM is the guarantor of this review.

\section{Funding}

This umbrella review will be conducted as part of a PhD studentship funded by the School of Medicine, University of St Andrews. The University of St Andrews played no role in the design and development of this protocol.

Availability of data and materials Not applicable. 


\section{Declarations}

Ethics approval and consent to participate

Not applicable.

\section{Consent for publication}

Not applicable.

\section{Competing interests}

The authors declare that they have no competing interests.

Received: 12 February 2021 Accepted: 21 October 2021

Published online: 01 November 2021

\section{References}

1. Javed Z, Sathyapalan T. Levothyroxine treatment of mild subclinical hypothyroidism: a review of potential risks and benefits. Therapeutic Adv Endocrinol Metab. 2016;7:12-23.

2. Surks MI, Ortiz E, Daniels GH, Sawin CT, Col NF, Cobin RH, et al. Subclinical thyroid disease: scientific review and guidelines for diagnosis and management. Jama. 2004;291:228-38.

3. Chubb S, Davis W, Inman Z, Davis T. Prevalence and progression of subclinical hypothyroidism in women with type 2 diabetes: the Fremantle Diabetes Study. Clin Endocrinol. 2005;62:480-6.

4. Schübel J, Feldkamp J, Bergmann A, Drossard W, Voigt K. Latent hypothyroidism in adults. Deutsches Ärzteblatt International. 2017:114:430.

5. Rodondi N, Den Elzen WP, Bauer DC, Cappola AR, Razvi S, Walsh JP, et al. Subclinical hypothyroidism and the risk of coronary heart disease and mortality. Jama. 2010;304:1365-74.

6. Razvi S, Weaver JU, Butler TJ, Pearce SH. Levothyroxine treatment of subclinical hypothyroidism, fatal and nonfatal cardiovascular events, and mortality. Arch Internal Med. 2012;172:811-7.

7. Flynn RW, Bonellie SR, Jung RT, MacDonald TM, Morris AD, Leese GP. Serum thyroid-stimulating hormone concentration and morbidity from cardiovascular disease and fractures in patients on long-term thyroxine therapy. J Clin Endocrinol Metab. 2010;95:186-93.

8. Kong WM, Sheikh MH, Lumb PJ, Freedman DB, Crook M, Doré CJ, et al. A 6-month randomized trial of thyroxine treatment in women with mild subclinical hypothyroidism. Am J Med. 2002;112:348-54.

9. Cooper DS, Biondi B. Subclinical thyroid disease. Lancet. 2012;379:1142-54.

10. Biondi B, Cooper DS. The clinical significance of subclinical thyroid dysfunction. Endocrine Reviews. 2008;29:76-131.

11. Vasileiou M, Gilbert J, Fishburn S, Boelaert K. Thyroid disease assessment and management: summary of NICE guidance. BMJ. 2020;368:m41.

12. UK National Guideline Centre. Thyroid disease: assessment and management. 2019.

13. Bekkering G, Agoritsas T, Lytvyn L, Heen A, Feller M, Moutzouri E, et al. Thyroid hormones treatment for subclinical hypothyroidism: a clinical practice guideline. BMJ. 2019;365:I2006.
14. Feller M, Snel M, Moutzouri E, Bauer DC, de Montmollin M, Aujesky D, et al. Association of thyroid hormone therapy with quality of life and thyroid-related symptoms in patients with subclinical hypothyroidism: a systematic review and meta-analysis. Jama. 2018;320:1349-59.

15. Baumgartner C, Blum MR, Rodondi N. Subclinical hypothyroidism: summary of evidence in 2014. Swiss Med Weekly. 2014;144:w14058.

16. Biondi B. Natural history, diagnosis and management of subclinical thyroid dysfunction. Best Pract Res Clin Endocrinol Metab. 2012;26:431-46.

17. Chrysant SG. The current debate over treatment of subclinical hypothyroidism to prevent cardiovascular complications. Int J Clin Practice. 2020;74:e13499.

18. Fusar-Poli P, Radua J. Ten simple rules for conducting umbrella reviews. Evidence-based Mental Health. 2018;21:95-100.

19. Centre for Reviews and Dissemination. PROSPERO International prospective register of systematic reviews: University of York; 2021 [cited 2021 15/01]. Available from: https://www.crd.york.ac.uk/prospero/.

20. Aromataris E, Fernandez R, Godfrey C, Holly C, Khalil H, Tungpunkom P. Chapter 10: Umbrella reviews. In: Aromataris E, Munn Z, editors. JBI Manual for Evidence Synthesis: JBI; 2020.

21. Shamseer $L$, Moher D, Clarke M, Ghersi D, Liberati A, Petticrew M, et al. Preferred Reporting Items for Systematic Review and Meta-Analysis Protocols (PRISMA-P) 2015: elaboration and explanation. Brit Med J. 2015;349:g7647. https://doi.org/10.1136/bmj.g7647.

22. Moher D, Shamseer L, Clarke M, Ghersi D, Liberati A, Petticrew M, et al. Preferred Reporting Items for Systematic Review and Meta-Analysis Protocols (PRISMA-P) 2015 statement. Systematic Reviews. 2015;4:1. https:// doi.org/10.1186/2046-4053-4-1.

23. The EndNote Team. EndNote. EndNote X9 ed. Philadelphia: Clarivate; 2013.

24. Veritas Health Innovation. Covidence systematic review software Melbourne, Australia2013. Available from: www.covidence.org.

25. Shea BJ, Grimshaw JM, Wells GA, Boers M, Andersson N, Hamel C, et al. Development of AMSTAR: a measurement tool to assess the methodological quality of systematic reviews. BMC Med Res Methodol. 2007;7:10.

26. Shea BJ, Reeves BC, Wells G, Thuku M, Hamel C, Moran J, et al. AMSTAR 2: a critical appraisal tool for systematic reviews that include randomised or non-randomised studies of healthcare interventions, or both. BMJ. 2017;358:j4008.

27. Lorenz RC, Matthias K, Pieper D, Wegewitz U, Morche J, Nocon M, et al. A psychometric study found AMSTAR 2 to be a valid and moderately reliable appraisal tool. J Clin Epidemiol. 2019;114:133-40.

28. Smith V, Devane D, Begley CM, Clarke M. Methodology in conducting a systematic review of systematic reviews of healthcare interventions. BMC Med Res Methodol. 2011;11:1-6.

29. Guyatt G, Oxman AD, Akl EA, Kunz R, Vist G, Brozek J, et al. GRADE guidelines: 1. Introduction-GRADE evidence profiles and summary of findings tables. J Clin Epidemiol. 2011;64:383-94.

\section{Publisher's Note}

Springer Nature remains neutral with regard to jurisdictional claims in published maps and institutional affiliations.
Ready to submit your research? Choose BMC and benefit from:

- fast, convenient online submission

- thorough peer review by experienced researchers in your field

- rapid publication on acceptance

- support for research data, including large and complex data types

- gold Open Access which fosters wider collaboration and increased citations

- maximum visibility for your research: over $100 \mathrm{M}$ website views per year

At BMC, research is always in progress.

Learn more biomedcentral.com/submissions 